\title{
Uma Tecnologia Assistiva Baseada na Semiótica Peirceana para a Educação Inclusiva de Crianças Surdas e Ouvintes
}

\author{
Denys Rocha ${ }^{1}$, Ig Ibert Bittencourt ${ }^{1}$, Rafael Amorim ${ }^{1}$, Patrícia Ospina ${ }^{2}$ \\ ${ }^{1}$ Instituto de Computação - Universidade Federal de Alagoas (UFAL) \\ Av. Lourival Melo Mota, s/n, Tabuleiro do Martins, CEP:57072-900, Maceió - AL \\ ${ }^{2}$ Departamento de Estatística - Universidade Federal de Pernambuco (UFPE) \\ Av. Prof. Moraes Rego, 1235 - Cidade Universitária, Recife - PE - CEP: 50670-901 \\ contato.denysrocha@gmail.com, \{ig.ibert, rafael\}@ic.ufal.br, \\ patespipadde.ufpe.br
}

\begin{abstract}
This paper proposes a model of inclusive education based on the Peircian Semiotics applied with technology, having as the main aim improve the communication process between deaf and listener children. In technology, signs are used as a way of learning. An experiment was carried out with children in a school in which the level of communication was observed during play. Subsequently the results were analysed in order to observe the quality of the communication. As main contribution of this paper, we have a semiotics model which supports alphabet learning of Portuguese and Libras(Brazilian Sign Language), as well as the improvement of the communication process between deaf and listener children.
\end{abstract}

\begin{abstract}
Resumo
Este trabalho propõe um modelo para educação inclusiva baseado na semiótica peirceana, sendo este incluído em uma tecnologia, tendo como objetivo melhorar o processo de comunicação entre crianças surdas $e$ ouvintes. Na tecnologia, utiliza-se o signo como forma de aprendizado. Foi realizado um experimento com crianças de uma escola, onde foi observado o nível de comunicação durante as brincadeiras. Posteriormente foram feitas análises dos resultados encontrados, afim de observar a qualidade dessa comunicação. Como principais contribuições deste trabalho, temos um modelo semiótico que apoia a aprendizagem do alfabeto das línguas Portuguesa e Libras, assim como a melhoria do processo de comunicação entre crianças surdas e ouvintes.
\end{abstract}

\section{Introdução}

Sabe-se que as pessoas surdas possuem necessidades específicas de interação e de inclusão social. Nas escolas atuais, por exemplo, elas são excluídas do direito de aprender, pois tais escolas privilegiam grupos de estudantes que atendem a um padrão de comunicação oral-auditivo, impedindo ou dificultando grupos que utilizem outro tipo de padrão, como a comunicação gesto-visual (FRIAS e MENEZES, 2008).

Nos últimos anos, alguns estudos vêm discutindo a proposta de um modelo de educação inclusiva que assegure a todos uma igualdade de oportunidades educativas, principalmente para pessoas com necessidades educacionais especiais (NEE) (FONSECA, 2004). 
Um dos principais obstáculos para uma educação inclusiva de qualidade é a falta de estímulo na comunicação entre crianças surdas e ouvintes, pois sem este estímulo não haverá inclusão de fato. Para que a inclusão realmente aconteça, deve haver recursos que assegurem os direitos de todos no processo educativo. Com a ausência desses recursos, a criança surda fica excluída de estabelecer comunicação com seus colegas ouvintes, assim como a criança ouvinte também perde de aprender e interagir com seu colega surdo. Percebe-se que a criação de um ambiente educacional inclusivo demanda de mais recursos que não estão disponíveis à escola, como intérpretes de língua de sinais, treinamento para os professores, acervos audiovisuais, etc. Neste caso, uma solução seria proporcionar uma educação inclusiva para crianças surdas e ouvintes num mesmo contexto utilizando tecnologias de informação e comunicação para reduzir a dependência de tais recursos.

Portanto, o presente trabalho tem por objetivo propor e discutir um modelo teórico incorporado a uma tecnologia para melhorar o processo de comunicação entre crianças surdas e ouvintes em fase de alfabetização, proporcionando uma educação mais inclusiva. Tal modelo e tecnologia são baseados nos conceitos da semiótica apresentada por Peirce (1977), sendo estendido pela inserção de um ciclo evolutivo de signos, no qual a criança aprende um novo conceito a partir de outro que a mesma já conhece. Tal extensão proporciona uma melhor interpretação de signos mais complexos, garantindo que uma criança exposta a este modelo possa compreender signos como grafemas, datilologia (alfabeto manual da língua de sinais) e sinais da Língua Brasileira de Sinais (Libras).

\section{Uma tecnologia assistiva para educação inclusiva}

Esta seção apresenta um modelo teórico baseado na Semiótica de Peirce, descrevendo o funcionamento dos processos de significação das crianças surdas e ouvintes referente ao aprendizado do alfabeto da língua portuguesa escrita e da Libras, com alguns de seus sinais. Logo em seguida, será mostrada uma instância do modelo gerado.

\subsection{O modelo baseado na semiótica peirceana}

Em um processo de alfabetização, as crianças ouvintes utilizam o canal auditivo e visual para receber determinada informação enquanto que as crianças surdas utilizam somente o campo gesto-espaço-visual. As duas falam línguas diferentes, ou seja, o Português e a Libras. Porém, ambas as línguas podem ser compreendidas através de signos apresentados em forma de sons, grafemas, imagens, símbolos ou expressões faciais e corporais. Desta forma, nesta seção serão apresentados dois modelos baseados na semiótica de Peirce (1977), entendendo as relações triádicas como base das construções de raciocínio das crianças, e o modelo referenciado por Eco (1986) para compreender quais os possíveis caminhos a serem trilhados num complexo rizoma de signos.

Considerando um possível caminho para a criança em seu processo de aprendizagem de grafemas, sons, datilologia, e sinais, criou-se um modelo onde sugerimos à criança uma sequência de relações, onde a mesma é levada a construir novos signos através de associações entre os signos apresentados. Esta sequência é apresentada em quatro relações complexas, onde relacionamos: (1) uma imagem de um objeto do mundo físico com o seu sinal em Libras; (2) a mesma imagem com um grafema, ou seja, letra escrita na Língua Portuguesa; (3) este grafema com a respectiva letra em datilologia/alfabeto manual da língua de sinais; e por fim, (4) todos os signos 
VI Congresso Brasileiro de Informática na Educação (CBIE 2017)

Anais dos Workshops do VI Congresso Brasileiro de Informática na Educação (WCBIE 2017)

anteriores aos seus respectivos Objetos, em uma forma de redundância.

No modelo apresentado (Figura 1), temos a relação entre todos os elementos. Portanto, temos como Representâmen ${ }^{1}$ desta relação todos os termos relacionados ao Objeto1 <OBJETO-1> e todos relacionados ao Objeto1 <OBJETO-2>, tendo como resultado Interpretante1 ( $<$ IMAGEM-1 $>\quad \mathrm{v} \quad<$ MULTIMEIO-1 $>\quad \mathrm{v} \quad<$ SOM-1 $>=$ $<$ OBJETO-1 $>) \supset(<$ IMAGEM-2 $>\mathrm{v}<$ MULTIMEIO-2 $>\mathrm{v}<$ SOM-2 $>=<$ OBJETO-2 $>)$. Assim sendo, nesta relação a mente interpretante pode relacionar uma letra escrita da Língua Portuguesa a um objeto físico e seus respectivos sons e sinais da Língua Brasileira de Sinais.

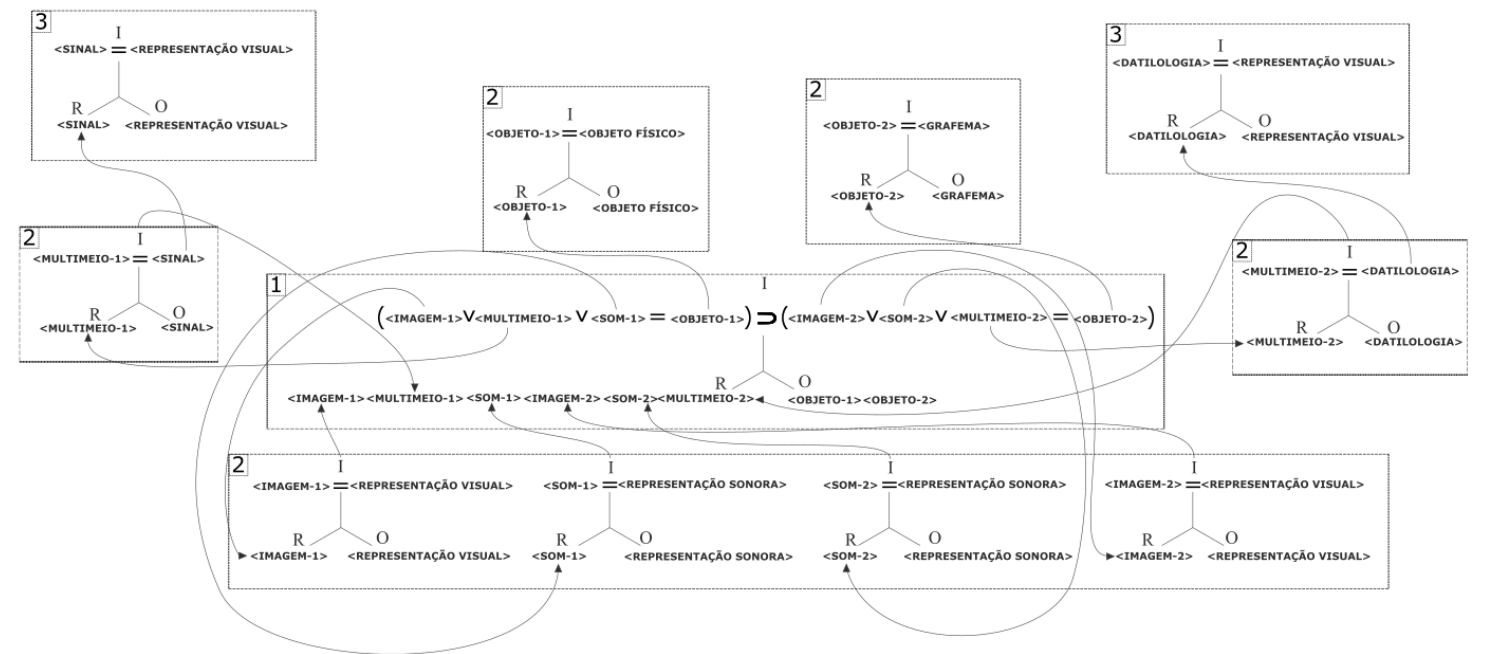

\section{Figura 1: Quarta relação - Visão completa da associação dos signos já vistos}

Nessa seção foi apresentado o modelo semiótico que explica um possível caminho de raciocínio que crianças surdas e ouvintes podem executar para relacionar elementos do mundo a sua volta com suas línguas faladas e sinalizadas, partindo do pressuposto que ambas significam e raciocinam através de signos, sendo estes comuns a ambas. Tal abordagem pode caracterizar o presente trabalho inclusivo, pois considera 0 processo de alfabetização bilíngue.

\subsection{Instância do modelo semiótico}

Para demonstrar os conceitos acima expostos, podemos citar como exemplo o aprendizado da relação entre o grafema B o objeto BOLA. Para um melhor entendimento do mesmo, continuaremos a utilizar a forma de legenda em camadas, ou seja, partindo das camadas com maior número de itens agregados, para suas unidades menores, ou relações simples.

Seguindo os mesmos princípios já mostrados no modelo teórico, chegamos a versão final de uma instância do modelo, representado na quarta e última relação (Figura 2), onde queremos relacionar todos os signos já apresentados com seus respectivos Objetos, $<$ BOLA $>$ e $<$ B $>$. Portanto como Representâmen desta relação (camada 1), temos os elementos $<$ IMAGEM $>$, $<$ MULTIMEIO $>$ e $<$ SOM $>$ que se referem ao Objeto 
$<$ BOLA $>$, e $<$ IMAGEM $>$, $<$ SOM $>$ e $<$ MULTIMEIO $>$ que se ligam ao objeto $<$ B $>$. Lembrando que o primeiro signo $<$ MULTIMEIO $>$ representa algum sinal em Libras, enquanto que o segundo representa uma letra do alfabeto manual da língua de sinais. $\mathrm{O}$ mesmo princípio se aplica ao primeiro signo <IMAGEM>, onde o mesmo representa algum objeto físico, enquanto que o segundo representa o desenho de uma letra/grafema da língua escrita. Note que todos os elementos do Representâmen se relacionam direta/indiretamente com os dois Objetos, isso nos leva ao Interpretante mais completo: $(<$ IMAGEM $>\mathrm{v}<$ MULTIMEIO $>\mathrm{v}<$ SOM $>=<$ BOLA $>) \supset(<$ MULTIMEIO $>\mathrm{v}$ $<$ SOM $>$ v $<$ IMAGEM $>=<$ B $>$ ).

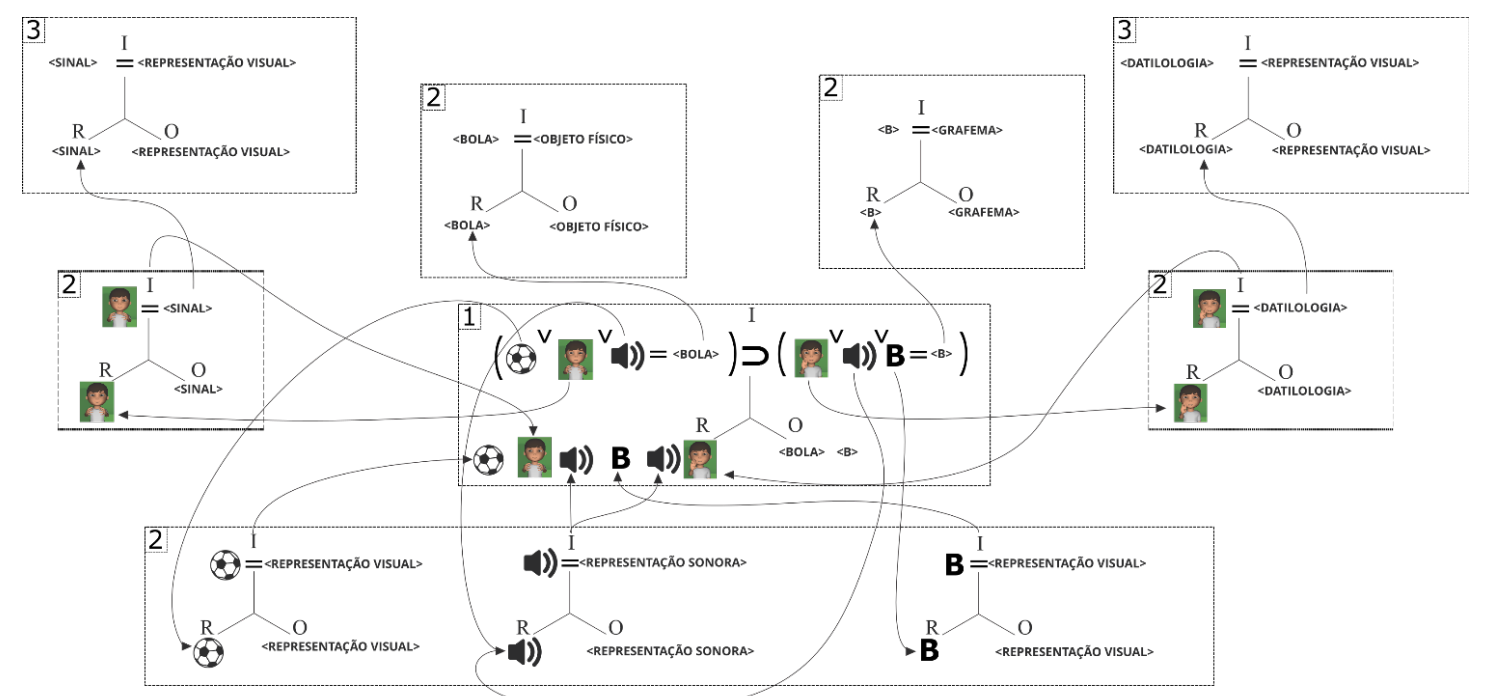
Figura 2: Quarta relação - Visão completa da associação dos
signos referente a relação BOLA $\supset$ B

Desta forma, concluímos a representação de uma instância do modelo semiótico que sugere um possível caminho de significação entre os Objetos $<$ BOLA $>$ e $<$ B $>$. O mesmo se aplica a todas as letras do alfabeto. Note que nesta relação todos os signos apresentados estão relacionados de alguma forma, tendo em vista que crianças surdas e ouvintes percebem quase os mesmos signos, pois o único incomum a ambas é som, não percebido pela criança surda, o que não invalida o modelo apresentado. Assim vimos uma possível maneira de iniciar o processo de alfabetização bilíngue, acessível e inclusivo, onde este pode ser viabilizado com o apoio de alguma tecnologia que supra a ausência nas escolas brasileiras de professores fluentes em língua de sinais.

\subsection{My Little Sign: Implementação do modelo semiótico numa tecnologia assistiva}

Visando sanar esta necessidade, foi desenvolvida uma tecnologia assistiva, que incorpora o modelo descrito anteriormente, possibilitando sua aplicabilidade num cenário real. Essa tecnologia assistiva é um software apresentado à criança como uma espécie de jogo, nomeado como "My Little Sign", onde a mesma aprende o conteúdo com o auxílio de um avatar 3D, conteúdo este referente ao alfabeto da Língua Portuguesa e da Libras, assim como alguns de seus sinais.

\subsubsection{Instância do modelo semiótico dentro da aplicação computacional}

Depois de encontrar uma forma de representação de raciocínio de pessoas com dois perfis diferentes, ou seja, surdos e ouvintes, implementou-se uma tecnologia que incorporasse tal modelo, tornando-o viável. Utilizando o mesmo exemplo da instância 
VI Congresso Brasileiro de Informática na Educação (CBIE 2017)

Anais dos Workshops do VI Congresso Brasileiro de Informática na Educação (WCBIE 2017)

do modelo semiótico, a relação entre "B" e "BOLA", podemos representá-lo dentro da tecnologia criada, como visto na Figura 3.

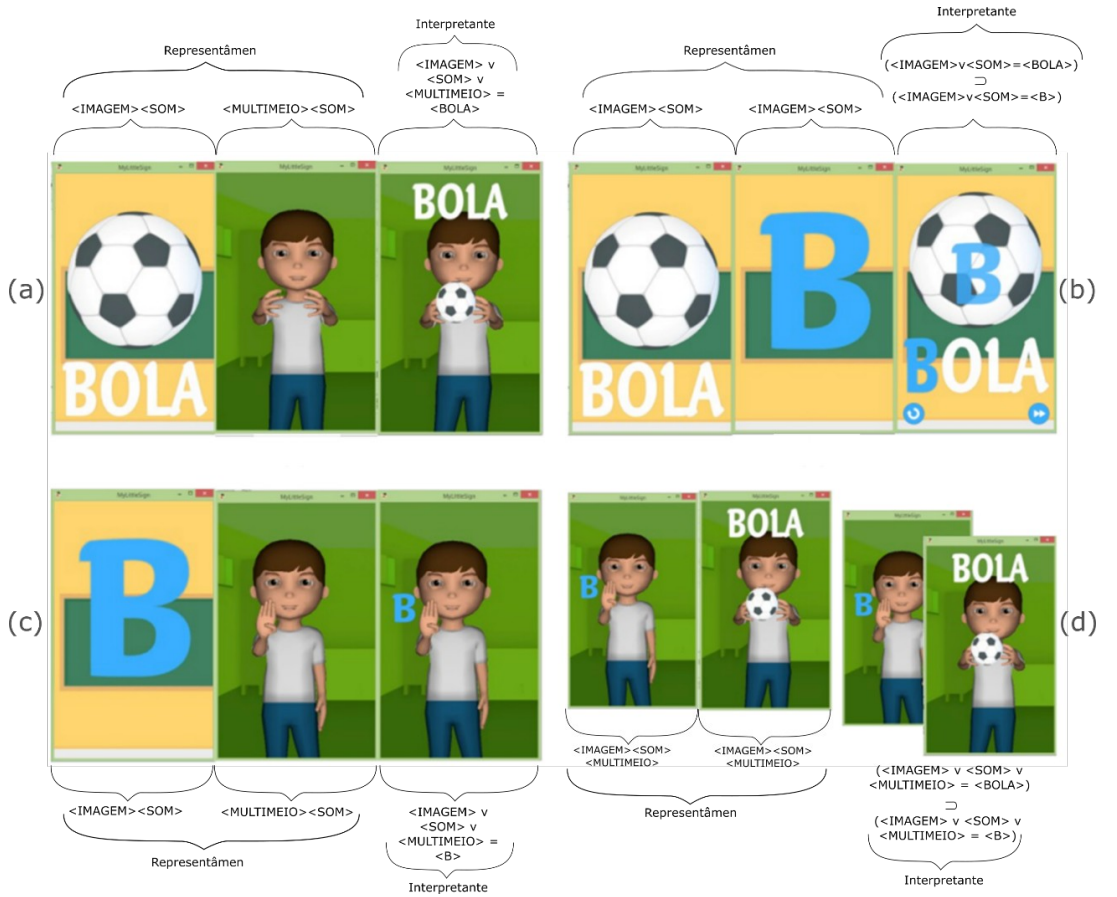

\section{Figura 3: Instância do modelo com a relação "B de BOLA" na tecnologia proposta}

Como visto na Figura 3, as quatro relações são apresentadas a criança utilizando elementos visuais e auditivos, seguindo a mesma sequência apontada no modelo semiótico e sua instância como o exemplo "B” de "BOLA” ou "BOLA" contém "B".

\section{Validação}

Para verificar a efetividade do modelo e aplicação computacional construída, foi realizado um quase-experimento com um grupo de alunos utilizando o jogo. Portanto, nesta seção serão abordadas questões relacionadas ao planejamento deste experimento, tais como principal questão de pesquisa, hipóteses, fatores e variáveis resposta, unidades experimentais e análise dos resultados.

\subsection{Questões de Pesquisa e Hipóteses}

Para criar um ambiente de educação inclusiva, no contexto de crianças surdas e ouvintes, faz-se necessária a presença da Língua Portuguesa e da Língua Brasileira de Sinais. Com a presença destas línguas a comunicação se torna mais fluida e semântica, caracterizando uma maior inclusão. Sendo assim, a principal questão de pesquisa relacionada a este quase-experimento objetiva comparar a eficiência da comunicação entre crianças surdas e ouvintes após a intervenção da tecnologia assistiva e professor ${ }^{2}$. P1 - A comunicação de crianças surdas e ouvintes apresenta diferença nas métricas de eficiência (uso de sinais, comunicação, uso de Libras, uso de Português, nenhum código, comunicação entre surdo e ouvinte, comunicação entre ouvintes) considerando a intervenção da tecnologia assistiva e professor?

2 A justificativa para a intervenção do professor está disponível e explicitada na versão completa do texto da dissertação do autor principal deste trabalho. 
VI Congresso Brasileiro de Informática na Educação (CBIE 2017)

Anais dos Workshops do VI Congresso Brasileiro de Informática na Educação (WCBIE 2017)

O que nos leva às seguintes hipóteses (Tabela 1):

\title{
Tabela 1: Hipóteses
}

H1-0: O uso de quaisquer sinais é igual independente da intervenção da tecnologia assistiva e professor.
H1-1: O uso de quaisquer sinais é diferente independente da intervenção da tecnologia assistiva e professor.

\begin{abstract}
H2-0: A comunicação entre todos os envolvidos é igual independente da intervenção da tecnologia assistiva e professor.

H2-1: A comunicação entre todos os envolvidos é diferente independente da intervenção da tecnologia assistiva e professor.

H3-0: O uso de sinais de Libras é igual independente da intervenção da tecnologia assistiva e professor.

H3-1: O uso de sinais de Libras é diferente independente da intervenção da tecnologia assistiva e professor.
\end{abstract}

H4-0: O uso de Português é igual independente da intervenção da tecnologia assistiva e professor. H4-1: O uso de Português é diferente independente da intervenção da tecnologia assistiva e professor.

H5-0: A comunicação entre surdo e ouvinte é igual independente da intervenção da tecnologia assistiva e professor.

H5-1: A comunicação entre surdo e ouvinte é diferente independente da intervenção da tecnologia assistiva e professor.

H6-0: A comunicação entre ouvintes é igual H6-1: A comunicação entre ouvintes é diferente independente da intervenção da tecnologia assistiva e professor. independente da intervenção da tecnologia assistiva e professor.

\subsection{Fatores e variáveis de resposta}

A variável independente ou fator utilizado no quase-experimento é a Intervenção utilizando a tecnologia assistiva e professor. De acordo com a Tabela 2, as variáveis resposta são:

\section{Tabela 2: Fatores}

\begin{tabular}{ll} 
Uso de sinais & $\begin{array}{l}\text { Se no período de observação analisado, houve algum tipo de sinal, sendo de } \\
\text { Libras ou algum sinal criado pela criança }\end{array}$ \\
\hline Uso de Libras & Se houve algum sinal de Libras praticado pela criança observada \\
\hline Uso de Português & Se houve a prática da Língua Portuguesa escrita ou falada pela criança observada \\
\hline Nenhum código & $\begin{array}{l}\text { Se não houve a presença de nenhuma língua padrão praticada pela criança no } \\
\text { período observado }\end{array}$ \\
\hline Comunicação & $\begin{array}{l}\text { Se houve alguma forma de comunicação entre a criança observada e quaisquer } \\
\text { outras }\end{array}$ \\
\hline $\begin{array}{l}\text { Comunicação entre surdo e e } \\
\text { ouvinte }\end{array}$ & $\begin{array}{l}\text { Se houve alguma forma de comunicação entre a criança (ouvinte) observada e } \\
\text { uma outra criança (surda) e vice-versa; }\end{array}$ \\
\hline Comunicação entre ouvintes & $\begin{array}{l}\text { Se houve alguma forma de comunicação entre a criança observada (ouvinte) e } \\
\text { uma outra criança (ouvinte). }\end{array}$ \\
\hline
\end{tabular}

\subsection{Unidades experimentais}

No nosso cenário, as unidades experimentais são os conjuntos das observações realizadas com um grupo de crianças com idades entre 7 e 10 anos, sendo 15 ouvintes e 1 surda, ou seja, são feitas 5 seções de observação do grupo, onde cada seção tem duração de 30 minutos. Na extração dos dados, cada criança é observada individualmente no período de 1 minuto, onde o fator deste experimento é aplicado para se obter as variáveis resposta já citadas anteriormente. Esse método de observação busca analisar as crianças no momento das brincadeiras, de acordo com Patern (1932) e Sager et al. (2003).

\subsection{Análise dos resultados}

Os resultados de cada métrica obtidos nas observações foram sumarizados na 
VI Congresso Brasileiro de Informática na Educação (CBIE 2017)

Anais dos Workshops do VI Congresso Brasileiro de Informática na Educação (WCBIE 2017)

ferramenta R e organizados para exibição gráfica através de histogramas e/ou boxplots, com o propósito de facilitar a comparação.

Quanto aos testes estatísticos, pretende-se realizar um teste para perceber a natureza dos dados, ou seja, se os mesmos provém de distribuição normal, para tal utilizaremos o Teste Anderson-Darling(ad) (BARBETTA et al, 2008). No caso percebeu-se que os dados não provinham de uma distribuição normal, portanto utilizamos testes não paramétricos, como o de Wilcoxon (BARBETTA et al, 2008). Todos os testes foram realizados com nível de confiança de 95\%. As mesmas conclusões puderam ser observadas através da criação de um modelo de regressão linear.

Nesta seção serão feitas as análises dos resultados obtidos com a realização do experimento controlado. Para tal, utilizou-se de um ferramental estatístico próprio para tratar e entender os dados, sendo o primeiro método o Teste Anderson-Darling(ad) (BARBETTA et al, 2008), que testa se os dados provem de uma população normal. Sendo assim:

Teste Anderson-Darling (ad): Seja $F(x)=P(X \leq x)$ a função de distribuição acumulada de uma população normal. Seja $G(x)$ a função de distribuição empírica dos dados (EDF em inglês), que pode ser definida como a função de distribuição acumulada das frequências relativas. O teste ad avalia se $G(x) \approx F(x)$. Então, nossas hipóteses são:

- $\quad H_{0}$ : os dados são provenientes de uma distribuição normal;

- $\quad H_{1}$ : os dados não são provenientes de uma distribuição normal;

Tabela 3: Teste de Normalidade Anderson Darling

\begin{tabular}{|c|c|c|}
\hline \multirow{2}{*}{ Variáveis } & \multicolumn{2}{|c|}{ P_Valores associados ao testes de normalidade Anderson-Darling } \\
\hline & Sem tecnologia & Com tecnologia e professor \\
\hline Sinal & $0.0000 * * *$ & $0.0005 * * *$ \\
\hline "Comunicação & $0.1436^{*}$ & $0.1094 *$ \\
\hline Português & $0.8269 *$ & 0.3764 \\
\hline Libras & $0.0000 * * *$ & $0.0007 * * *$ \\
\hline Português/Libras & $0.0000 * * *$ & $0.0000 * * *$ \\
\hline Nenhum Código $^{3}$ & $0.9611^{*}$ & $0.4880^{*}$ \\
\hline Surdo & $0.0000 * * *$ & $0.0002 * * *$ \\
\hline Ouvinte & $0.5152 *$ & $0.2325 *$ \\
\hline Surdo/Ouvinte & $0.0000 * * *$ & $0.0000 * * *$ \\
\hline
\end{tabular}

Nota-se a partir da Tabela 3 que mais da metade dos p_valores são menores que $5 \%$ indicando que rejeitamos a hipótese de normalidade para essas variáveis ao nível de $5 \%$. Neste sentido podemos utilizar testes não paramétricos para comparar se as variáveis da amostra sem tecnologia e amostra com tecnologia e professor são provenientes da mesma distribuição de probabilidade. Neste caso, vamos utilizar o teste Wilcoxon (BARBETTA et al, 2008) para duas populações. As hipóteses do teste então descritas abaixo, em que $F_{X}$ representa a distribuição de probabilidades da variável aleatória $\mathrm{X}$ e $\mathrm{F}_{\mathrm{Y}}$ representa a distribuição de probabilidades da variável aleatória $\mathrm{Y}$. O

3 Código aqui se refere a utilização de uma Língua padrão 
VI Congresso Brasileiro de Informática na Educação (CBIE 2017)

Anais dos Workshops do VI Congresso Brasileiro de Informática na Educação (WCBIE 2017)

teste é realizado considerando um nível de significância $\alpha=0.05=5 \%$.

Como nosso interesse é testar a equivalência das populações referentes às amostras sem tecnologia e com tecnologia e professor, teremos hipóteses do tipo:

$$
\begin{aligned}
& \mathrm{H}_{0}: \mathrm{F}_{\text {UsoDeSinal-SemTecnologia }}=\mathrm{F}_{\text {UsoDeSinal-ComTecnologiaProfessor. }} \\
& \mathrm{H}_{1}: \mathrm{F}_{\text {UsoDeSinal-SemTecnologia }} \neq \mathrm{F}_{\text {UsoDeSinal-ComTecnologiaProfessor. }}
\end{aligned}
$$

\begin{tabular}{|c|c|}
\hline \multicolumn{2}{|c|}{ P_Valores - Teste de Wilcoxon - Grupo Sem Tecnologia e Grupo com Tecnologia e Professor } \\
\hline Uso de Sinais & $0.002364 * *$ \\
\hline Comunicação & $0.558600 *$ \\
\hline Uso de Português & $0.738900^{*}$ \\
\hline Uso do Libras & $0.001366^{* *}$ \\
\hline $\begin{array}{c}\text { Nenhum Código } \\
\end{array}$ & $0.209400 *$ \\
\hline Comunicação entre Surdo e Ouvinte & $0.818700^{*}$ \\
\hline Comunicação entre Ouvintes & $0.949100^{*}$ \\
\hline
\end{tabular}

Tabela 4: Teste de Wilcoxon

Com base na Tabela 4, notamos que apenas as amostras Uso de Sinais - Sem e Com Tecnologia (uso de sinais pelos alunos) e as amostras Uso de Libras - Sem e Com Tecnologia (uso de Libras entre os alunos) são consideradas provenientes de populações diferentes. Tendo os P-Valores iguais a 0.002364 e 0.001366 , respectivamente e menores que 0.05 , logo podemos concluir com base nestas amostras que o uso de sinais de Libras e sinais criados entre as crianças antes do uso da tecnologia e após o uso da tecnologia e auxílio do professor são diferentes estatisticamente ao nível de 5\%. A mesma conclusão é válida quanto ao uso de somente sinais de Libras.

A seguir realizaremos uma análise estatística descritiva dos dados baseada nos gráficos: histogramas, boxplots e qqplots. Na Figura 4 são apresentados os histogramas das amostras de comunicação usando sinais e usando Libras, sem o uso da tecnologia e com o uso da tecnologia e auxílio do professor. Assim, essas figuras confirmam os resultados dos testes acima, ou seja, que existe diferença expressiva entre os grupos em tecnologia e com tecnologia e professor, para as variáveis sinais e Libras.
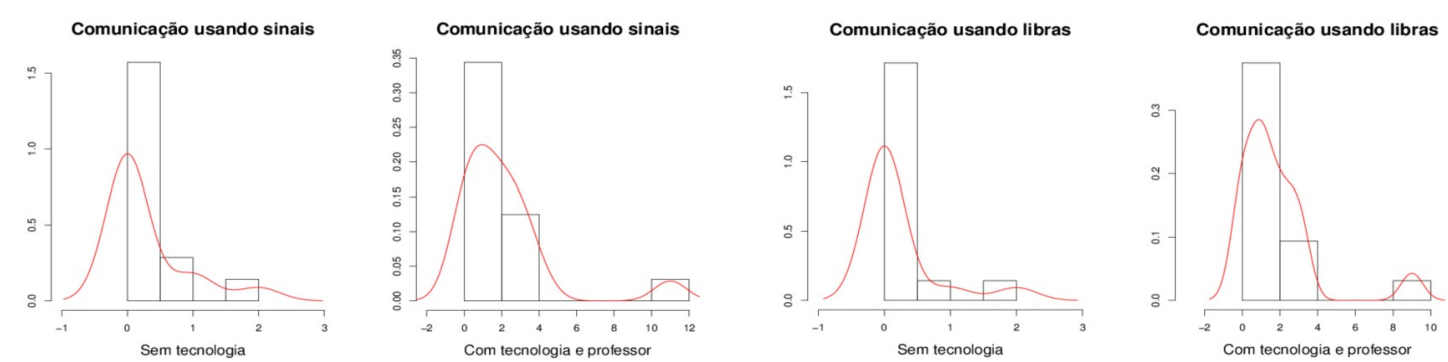

Figura 4: Histograma de comparação entre as variáveis "sinais" e
"Libras" com e sem "tecnologia e professor"

Em seguida apresentamos os BoxPlots das variáveis envolvidas no estudo (Figura 5), os quais confirmam as conclusões acima, ou seja, é evidente que a quantidade de Sinais e de Libras dos grupos sem e com tecnologia é consideravelmente diferente, sendo que as quantidades de sinais e Libras são muito mais expressivas para o 
VI Congresso Brasileiro de Informática na Educação (CBIE 2017)

Anais dos Workshops do VI Congresso Brasileiro de Informática na Educação (WCBIE 2017)

grupo com tecnologia e professor.
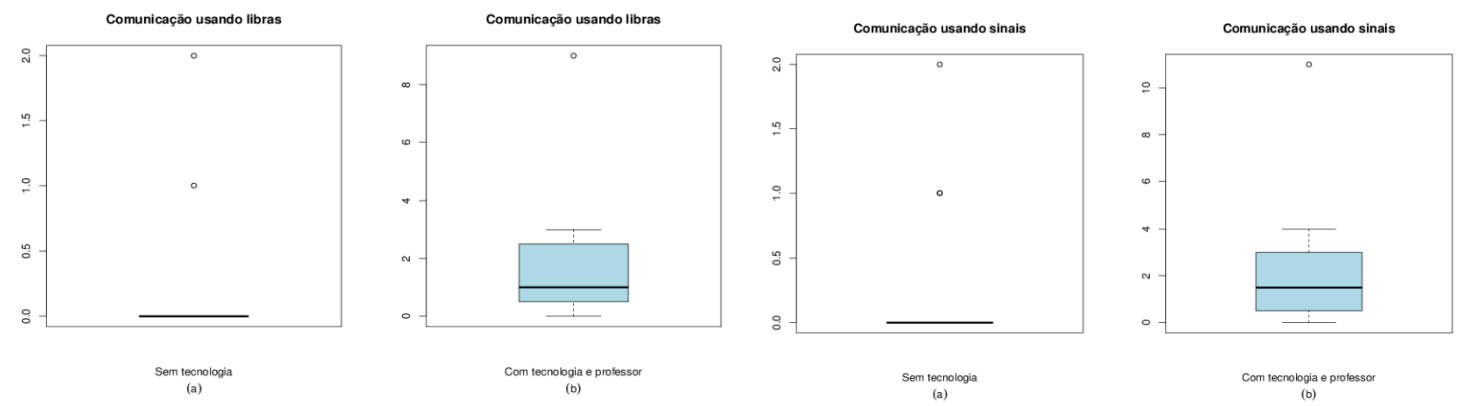

Figura 5: BoxPlots das variáveis "sinais" e "Libras" com e sem "tecnologia e professor"

Em relação aos QQplots (Figura 6) espera-se uma aderência dos pontos à reta diagonal, quando a variável aleatória apresenta distribuição normal. Se essa distribuição normal for a padrão, isto é, com média "0" e variância "1", então a reta vermelha deve ser a primeira bissetriz. Com base na Figura 6, é possível perceber que nenhum dos dois comportamentos esperados acontece para as variáveis relacionadas, evidenciado que a suposição de normalidade para estas variáveis, pode ser contestada, até porque os dados são discretos, o que também fica bem evidente nos Qqplots.
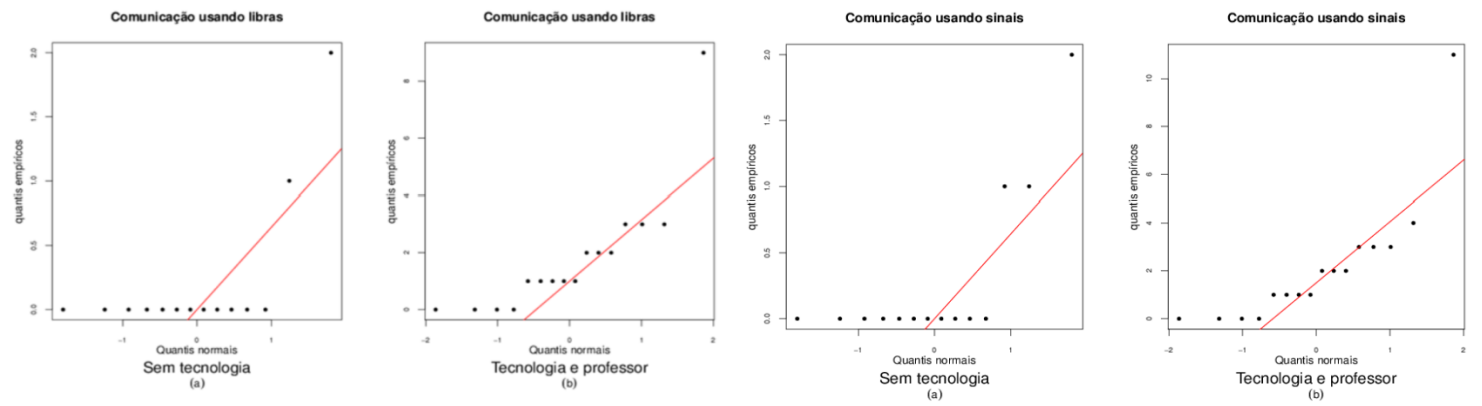

Figura 6: QQPlots das variáveis "sinais" e "Libras" com e sem "tecnologia e professor"

Concluímos que o processo de comunicação entre crianças ouvintes e a surda melhorou a nível quantitativo, com o aumento do uso de sinais, e qualitativo, pois com o uso destes sinais, as crianças possuíam um vocabulário maior em comum, tornando o processo comunicativo mais semântico.

\section{Conclusão}

O foco deste trabalho foi investigar uma forma de criar um ambiente de sala de aula mais inclusivo, considerando crianças surdas e ouvintes aprendendo simultaneamente suas línguas nativas. O modelo e tecnologia gerados consistiram-se em forma final de um jogo, onde a criança pode aprender conteúdos relacionados ao alfabeto da Língua Portuguesa e da Libras interagindo com um personagem 3D.

Este trabalho contribui para as seguintes áreas de conhecimento: (i) Informática na Educação, pois há uma forte tendência na área em observar como a computação e os meios informáticos podem melhorar a qualidade da Educação. (ii) Interação Humano Computador (IHC), pois gera um artefato teórico e computacional que visa melhorar a 
relação entre pessoas através da tecnologia; (iii) Tecnologias Assistivas, pois advem do princípio do termo Acessibilidade, onde o artefato computacional torna acessível as oportunidades para todos, levando em consideração suas reais necessidades; e (iv) Educação Inclusiva, de acordo com o conceito de Fonseca (2004) e outros autores da mesma linha, pois o objetivo principal deste trabalho é dar acesso à educação de forma equitativa para crianças surdas e ouvintes, melhorando sua comunicação e consequentemente sua inclusão.

Para verificar se esta proposta de solução teria eficácia, um quase-experimento foi executado, afim de colher e analisar dados das interações de crianças ouvintes e surdas após o uso da tecnologia proposta. Para tal, verificou-se se os dados eram provenientes de uma população normal utilizando o teste Anderson-Darling, o que constatou-se a rejeição de normalidade. Percebendo isto, utilizou-se testes não paramétricos para observar se os dados proviam de uma mesma distribuição de probabilidade. Para tal, utilizou-se o teste de Wilcoxon para as populações: sem tecnologia (crianças brincando sem intervenção); e com tecnologia e professor (com intervenção da tecnologia proposta e auxílio do professor). Após o teste, percebeu-se que os dados provinham de populações diferentes.

Com os resultados obtidos, vimos através dos histogramas e BoxPlots que há uma maior concentração de sinais de Libras e sinais criados por crianças no grupo "com tecnologia e professor", o que fortalece a ideia que a proposta de solução deste trabalho é válida. Contudo, para verificar os mesmos resultados, foi criado e aplicado um modelo de regressão ${ }^{4}$, utilizando a distribuição de Poisson. Ao final das análises percebeu-se que houve mudança significativa no nível de comunicação entre as crianças participantes, utilizando o Português a Libras. Tais mudanças eram percebidas através do uso mais frequente de sinais de Libras entre alunos ouvintes e a colega surda.

\section{Referências}

ECO, U. "Semiotics and the Philosophy of Language". Indiana University Press, 1986.

Espinheira, P.L.; Ferrari, S.L.P.; Cribari-Neto, F., "On beta regression residuals", Journal of Applied Statistics, v.35, p407-419, 2008.

FONSECA, V. da. "Tendências Futuras da Educação Inclusiva”. In: STOBÄUS, C. D; MOSQUERA, J. J. M. (Orgs). Educação Especial: em direção à educação inclusiva. 2 ed. Porto Alegre: EDIPUCRS, 2004, p. 41-63.

FRIAS, E. M. A.; MENEZES, M. C. B. "Inclusão escolar do aluno com necessidades educacionais especiais: contribuições ao professor do Ensino Regular". PDE, FAFIPA， 2008， http://www.diaadiaeducacao.pr.gov.br/portals/pde/arquivos/14628.pdf. Acesso em: 01 de junho, 2015.

PARTEN, M. B. "Social participation among pre-school children". The Journal of Abnormal and Social Psychology, v. 27, n. 3, p. 243, 1932.

PEIRCE, C.S. "Semiótica", trad. de Teixeira Coelho. 4 ed. $1^{\text {a }}$ Reimpressão. São Paulo: Perspectiva, 2012.

BARBETTA, A.P., REIS, M.M., BORNIA, A.C. "Estatística Para Cursos De Engenharia e Informática”. 2. ed. São Paulo: Atlas, 2008.

4 Disponível na versão completa do texto da dissertação do primeiro autor 